\title{
Association of serum irisin concentrations with the presence and severity of obstructive sleep apnea syndrome
}

\author{
Yanli $\mathrm{Li}^{1,2 \#} \mid$ Xueqin $\mathrm{Li}^{3 \#} \mid$ Dejun Sun ${ }^{2} \mid$ Shaoxi Cai ${ }^{1}$
}

${ }^{1}$ Department of Respiratory and Critical Care Medicine, Southern Hospital, Southern Medical University, Guangzhou, China

${ }^{2}$ Department of Respiration, Inner Mongolia People's Hospital, Hohhot, China

${ }^{3}$ Inner Mongolia Medical University, Hohhot, China

\section{Correspondence}

Shaoxi Cai, Department of Respiration, Southern Hospital, Southern Medical University, Guangzhou, China.

Email: caisxaoxi@yeah.net
Objective: Obesity is involved in the pathogenesis of obstructive sleep apnea syndrome (OSAS). Irisin, a recently discovered myokine, protects the mice from obesity. This study aims to determine the association of serum irisin concentrations with the presence and severity of OSAS.

Methods: This cross-sectional investigation was performed in 165 male OSAS patients and 98 healthy male subjects. Serum irisin concentrations were assessed using an enzyme-linked immunosorbent assay kit.

Results: The serum irisin concentrations of OSAS patients significantly decreased compared with the healthy controls $(P<.001)$. Multivariable logistic regression analysis indicated that serum irisin concentrations were an independent determinant of OSAS (OR .971, $95 \% \mathrm{Cl} .960$ to $.981 ; P<.001$ ). Serum irisin concentrations were significantly reduced among patients with severe OSAS compared with patients with mild and moderate OSAS $(P<.001$ and $P=.010$, respectively). Spearman correlation analysis revealed that serum irisin concentrations were inversely correlated with OSAS severity $(r=-.327, P<.001)$.

Conclusion: Decreased serum irisin concentrations are associated with the presence and severity of OSAS.

KEYWORDS

inflammation, irisin, obesity, obstructive sleep apnea syndrome

\section{1 | INTRODUCTION}

Obstructive sleep apnea syndrome (OSAS) is characterized by the cessation of respiration during sleep due to the obstruction of the upper airways. ${ }^{1}$ OSAS is a highly prevalent disease affecting approximately $4 \%$ of males and $2 \%$ of females in the adult population. ${ }^{2}$ OSAS contributes to increased morbidity and mortality of humans, particularly from cardiovascular diseases. ${ }^{3}$ The exact mechanism of OSAS is unclear. Recent investigations focused on the importance of obesity in the pathogenesis of OSAS. Furthermore, obesity is considered as the most important risk factor of OSAS. ${ }^{4}$ At least two out of every three OSAS patients are estimated to be obese. ${ }^{5}$ Subjects with a body mass

\#These two authors contribute equally to this article.

Source of Funding: No funding index (BMI) of more than $29 \mathrm{~kg} / \mathrm{m}^{2}$ exhibited a 10 -fold increased risk for OSAS. ${ }^{5}$ Inflammation is also involved in the mechanism of OSAS. ${ }^{6}$ Lots of inflammatory molecules were demonstrated to be correlated with OSAS. ${ }^{6}$

Irisin, an exercise-induced myokine, is secreted into the circulation. Recently, irisin is secreted not only by muscle but also by adipose tissues. Therefore, irisin is also considered as an adipokine. ${ }^{7,8}$ Elevated irisin levels in the circulation increased the energy expenditure in mice with no changes in movement or food intake. ${ }^{9}$ Irisin reverses diet-induced obesity and diabetes by stimulating thermogenesis in mice by promoting the proliferation of brown adipocyte-like cells. ${ }^{10}$ In humans, serum irisin levels were found to be negatively correlated with BMI. ${ }^{7}$ Serum irisin concentrations were significantly lower in obese subjects than lean ones. ${ }^{11}$ In addition, irisin restored ox-LDL-induced human umbilical vein endothelial cell dysfunction by reducing the levels of inflammatory 
genes. ${ }^{12}$ Therefore, irisin is speculated to mediate the pathogenesis of OSAS through its anti-obesity and anti-inflammation effects.

This study aims to determine whether serum irisin concentrations are correlated with the presence and severity of OSAS.

\section{2 | MATERIALS AND METHODS}

\section{1 | Patients}

This study consisted of 165 male patients newly diagnosed with OSAS through polysomnography (PSG). The exclusion criteria included psychiatric disorders, history of alcohol and drug abuse, and systemic illnesses, such as cardiovascular, cerebrovascular, pulmonary, or neuromuscular disease. Ninety-eight male healthy subjects who went on a medical check-up in our hospital were recruited for the control group. The controls were matched with OSAS patients in age and BMI, and diagnosed without OSAS using PSG. Subjects with systemic illnesses such as heart failure, chronic renal failure, and chronic obstructive pulmonary disease were excluded from the control group. The study was designed according to the ethics guidelines of the Helsinki Declaration and was approved by the Institutional Research Ethics Board of our hospital. All patients provided a written informed consent for their participation in this study.

\section{2 | Sleep study}

Full PSG monitoring was performed by the Compumedics E-series Sleep System (Compumedics Sleep: Melbourne, Australia). Apnea was defined as the cessation of breathing for at least 10 seconds, whereas hypopnea referred to the $50 \%$ decrease in nasal airflow with at least $4 \%$ decrease in oxygen saturation. The apnea-hypopnea index (AHI) was the average frequency of apnea and hypopnea in 1 hour. ${ }^{13}$ Subjects with $\mathrm{AHI}<5$ were included in the control group. The diagnosis of OSAS was based on $\mathrm{AHI} \geq 5$ events/ $h$ which was further subdivided into mild ( $5 \leq \mathrm{AHI}<15$ events $/ \mathrm{h}$ ), moderate ( $15 \leq \mathrm{AHI}<30$ events $/ \mathrm{h}$ ), and severe $(\geq 30 \text { events } / \mathrm{h})^{13}$

\section{3 | Measurements}

During the initial examinations, the height, weight, and blood pressure of the subjects were measured. Venous blood was collected after at least a 10-hour fasting. The blood specimen was then centrifuged and stored at $-80^{\circ} \mathrm{C}$ until examination. Serum irisin concentrations were measured using an enzyme-linked immunosorbent assay (\#EK-067-29, Phoenix Pharmaceuticals, Inc., Belmont, CA, USA) [coefficient of variations (CVs) of intra assay: $5 \%-7 \%$; $C V$ s of inter assay: $12 \%-15 \%$; detecting range: $0.1-1000 \mathrm{ng} / \mathrm{mL}$; linear range: $1.29-27.5 \mathrm{ng} / \mathrm{mL}$ ]. The serum was diluted to a suitable concentration to match the best linear range of the kit.

\section{4 | Statistical analysis}

Power and sample size calculators was utilized to calculate the sample size ( $\alpha$ of .05 and power of 90\%). A minimum of 21 subjects in the OSAS and control groups are necessary to obtain the difference in serum irisin concentrations. Data are presented as means \pm SD or median (interquartile range). Data normality was analyzed using the Kolmogorov-Smirnov test. The characteristics of OSAS patients were compared with healthy controls through unpaired $t$-test (normally distributed data) or Mann-Whitney U-test (not normally distributed data). Univariate analysis was performed and the variables with a $P<.10$ were then entered into a backward stepwise multivariate logistic regression model to calculate the odds ratio values (OR) and $95 \%$ confidence intervals $(\mathrm{Cl})$ for the presence of OSAS. KruskalWallis test was used to compare the differences in serum irisin concentrations among patients with mild, moderate, and severe OSAS. Afterwards, Mann-Whitney U-test was performed to compare the subgroup differences in serum irisin concentrations among patients with mild, moderate, and severe OSAS. The correlation of serum irisin concentrations with the severity of OSAS and the correlation between serum irisin and other parameters were analyzed using Spearman correlation analysis. Statistical significance was accepted at a $P$-value of $<.05$.

\section{3 | RESULTS}

\section{1 | Baseline clinical characteristics}

Patients with OSAS demonstrated elevated levels of homeostasis model assessment-insulin resistance (HOMA-IR), total cholesterol (TC), low-density lipoprotein cholesterol (LDL-C), and AHI compared with healthy controls (Table 1).

\subsection{Serum irisin concentrations in OSAS patients}

Serum irisin concentrations were significantly decreased in OSAS patients compared with healthy controls $(P<.001$; Table 1$)$. Simple logistic regression analysis indicated that HOMA-IR (OR 2.844, 95\% $\mathrm{Cl} 1.915$ to 4.223 ; $P<.001$ ), TC (OR $1.323,95 \% \mathrm{Cl} 1.044$ to 1.676; $P=.020$ ), LDL-C (OR 1.523, 95\% Cl 1.092 to 2.213; $P=.013$ ), and serum irisin (OR .973, 95\% Cl .964 to .983; $P<.001$ ) showed a trend toward an association with the presence of OSAS (Table 2). All these parameters were then entered into a multivariate logistic regression model. The adverse association of serum irisin concentrations with the presence of OSAS persisted (OR .971, 95\% Cl .960 to .981; $P<.001$ ) (Table 2).

\subsection{Serum irisin concentrations with the severity of OSAS}

Patients with severe OSAS reported significantly lower serum irisin concentrations compared with those with mild and moderate OSAS $(P<.001$ and $P=.010$, respectively) (Figure 1). Furthermore, decreased serum irisin concentrations were observed among moderate patients compared with mild patients ( $P=.014$; Figure 1$)$. Spearman correlation analysis suggested the negative correlation of serum irisin concentrations with OSAS severity $(r=-.327, P<.001)$. 
TABLE 1 Clinical and biochemical characteristics of OSAS patients and healthy controls

\begin{tabular}{|c|c|c|c|}
\hline & OSAS patients & Control & $P$-value \\
\hline Age (y) & $54.36 \pm 6.94$ & $54.72 \pm 5.99$ & .669 \\
\hline $\mathrm{SBP}(\mathrm{mm} \mathrm{Hg})$ & $144.07 \pm 17.35$ & $142.33 \pm 12.36$ & .385 \\
\hline $\mathrm{DBP}(\mathrm{mm} \mathrm{Hg})$ & $88.73 \pm 9.73$ & $88.21 \pm 7.50$ & .654 \\
\hline $\mathrm{TC}(\mathrm{mmol} / \mathrm{L})$ & $5.50 \pm 1.10$ & $5.16 \pm 1.16$ & .019 \\
\hline $\mathrm{TG}(\mathrm{mmol} / \mathrm{L})$ & $2.06 \pm 0.57$ & $1.85 \pm 0.62$ & .133 \\
\hline LDL-C (mmol/L) & $3.55 \pm 0.77$ & $3.30 \pm 0.86$ & .012 \\
\hline $\mathrm{HDL}-\mathrm{C}$ (mmol/L) & $1.15 \pm 0.21$ & $1.16 \pm 0.24$ & .765 \\
\hline
\end{tabular}

OSAS, obstructive sleep apnea syndrome; BMI, body mass index; SBP, systolic blood pressure; DBP, diastolic blood pressure; HOMA-IR, homeostasis model assessment of insulin resistance; TC, total cholesterol; TG, triglycerides; LDL-C, low-density lipoprotein cholesterol; HDL-C, high-density lipoprotein cholesterol; $\mathrm{AHI}$, apnea-hypopnea index.

TAB LE 2 Logistic regression Analysis for the presence of OSAS

\begin{tabular}{|c|c|c|c|c|}
\hline & \multicolumn{2}{|l|}{ Simple regression } & \multicolumn{2}{|l|}{ Multiple regression } \\
\hline Age (y) & $0.992(0.955-1.030)$ & .667 & & \\
\hline BMI $\left(\mathrm{kg} / \mathrm{m}^{2}\right)$ & $1.040(0.966-1.119)$ & .298 & & \\
\hline $\mathrm{SBP}(\mathrm{mm} \mathrm{Hg})$ & $1.007(0.991-1.024)$ & .384 & & \\
\hline $\mathrm{TC}(\mathrm{mmol} / \mathrm{L})$ & $1.323(1.044-1.676)$ & .020 & $1.441(1.098-1.890)$ & .008 \\
\hline TG (mmol/L) & $1.213(0.941-1.565)$ & .137 & & \\
\hline LDL-C (mmol/L) & $1.523(1.092-2.213)$ & .013 & $1.548(1.059-2.261)$ & \\
\hline $\mathrm{HDL}-\mathrm{C}$ (mmol/L) & $1.242(0.461-3.351)$ & .668 & & .024 \\
\hline
\end{tabular}

Abbreviations as Table 1.

\subsection{The correlation of serum irisin with other parameters}

Table 3 shows the Spearman correlation analysis results indicating the correlation of serum irisin with BMI ( $r=-.230, P=.003)$, LDL-C $(r=-.245, P=.001)$, and AHI $(r=-.302, P<.001)$.

\section{4 | DISCUSSION}

Obesity is considered a key risk factor for the development and progression of OSAS. ${ }^{14}$ Obese or severely obese patients showed approximately twice the prevalence of OSAS compared with normal-weight adults. In addition, mild OSAS patients experienced a $10 \%$ weight gain demonstrated a sixfold increased risk of progressing OSAS severity, whereas a $10 \%$ weight loss contributed to over $20 \%$ improvement of OSAS severity. ${ }^{15}$ The exact mechanism of obesity leading to the development and progression of OSAS remains unclear. Obesity possibly results in and worsens OSAS by fat deposition at specific sites. ${ }^{16}$ Increased fat tissues in the neck may have additional effects on the collapsibility of the upper airway and increase OSAS susceptibility. ${ }^{17}$ In addition, many genetic polymorphisms may positively affect obesity and OSAS, and may be interrelated in the development of these conditions. $^{18}$

Irisin stimulates the uncoupling protein 1 expression in white adipose cells in culture and in vivo. ${ }^{9}$ Irisin production is induced by exercise in mice and humans. ${ }^{9}$ In addition, elevated serum irisin concentrations help increase energy expenditure and weight loss in mice. ${ }^{9}$ Huh et al. ${ }^{19}$ reported that irisin caused the uncoupling protein 1 upexpression and consequently increased adipocyte energy expenditure and reduced lipid accumulation in human adipocytes. In addition, irisin inhibits preadipocyte differentiation, suggesting its additional role in 


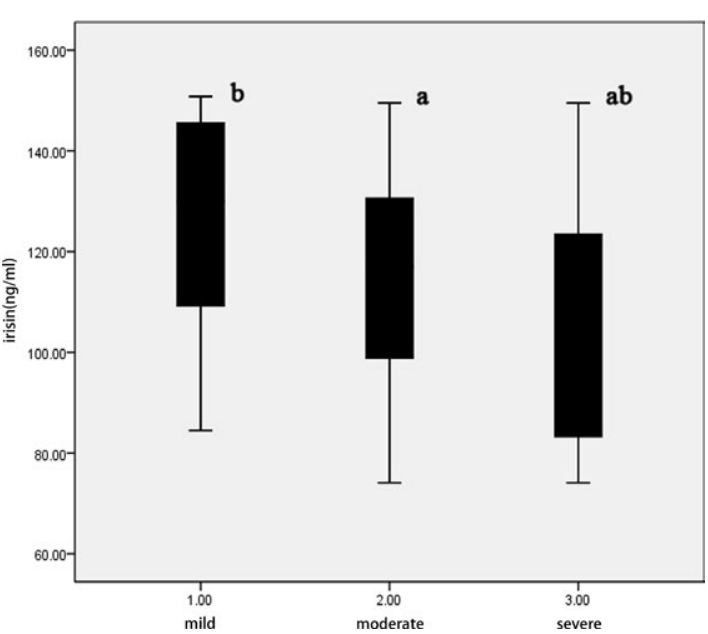

FIGURE 1 Serum irisin concentrations in mild, moderate, and severe obstructive sleep apnea syndrome (OSAS) patients. Severe OSAS patients showed significantly lower serum irisin concentrations compared with mild and moderate OSAS patients. Furthermore, there are decreased serum irisin concentrations in moderate patients compared with mild patients. ${ }^{a} P<.05$ vs patients with mild OSAS; ${ }^{\mathrm{b}} P<.05$ vs. patients with moderate OSAS

TABLE 3 The correlation between irisin and other clinical parameters

\begin{tabular}{|lcc|}
\hline Parameters & $r$ & P-value \\
\hline Age $(\mathrm{y})$ & -.099 & .206 \\
\hline $\mathrm{BMI}\left(\mathrm{kg} / \mathrm{m}^{2}\right)$ & -.230 & .003 \\
\hline $\mathrm{SBP}(\mathrm{mm} \mathrm{Hg})$ & -.047 & .545 \\
\hline $\mathrm{DBP}(\mathrm{mm} \mathrm{Hg})$ & -.033 & .677 \\
\hline $\mathrm{HOMA-IR}$ & .104 & .112 \\
\hline $\mathrm{TC}(\mathrm{mmol} / \mathrm{L})$ & .018 & .823 \\
\hline $\mathrm{TG}(\mathrm{mmol} / \mathrm{L})$ & .047 & .552 \\
\hline $\mathrm{LDL}-\mathrm{C}(\mathrm{mmol} / \mathrm{L})$ & -.245 & .001 \\
\hline $\mathrm{HDL}-\mathrm{C}(\mathrm{mmol} / \mathrm{L})$ & .072 & .359 \\
\hline AHI & -.302 & $<.001$ \\
\hline
\end{tabular}

Abbreviations as Table 1.

suppressing fat mass. ${ }^{19}$ Obese children showed increased circulating irisin levels after 1 year of exercise and lifestyle intervention. ${ }^{20}$ All these findings demonstrated the important role of irisin in obesity. Obesity is a clear risk factor for OSAS. Therefore, irisin is hypothesized to have a protective role in the pathogenesis of OSAS by inhibiting obesity development. However, other studies reported contradicting results. In humans, serum irisin levels were found to be negatively correlated with $\mathrm{BMI},{ }^{7,21}$ whereas other investigators reported that circulating irisin levels were positively correlated with BMI. ${ }^{22-24}$ These inconsistent data may be caused by the different conditions or diseases among the subjects, as well as the differences in the populations, or the assays applied in the studies.

The OSAS mechanism involves inflammation. lirisin is demonstrated to have an anti-inflammatory role. The FNDC4 is a secreted factor sharing high homology with FNDC5. The FNDC4 administration in bone marrow-derived macrophages helped reduce phagocytosis, increase cell survival, and reduce proinflammatory chemokine expression. ${ }^{25}$ Irisin can provide protection against diabetic retinopathy through the potential effects on anti-interleukin-17A. ${ }^{26}$ Irisin restored ox-LDL-induced human umbilical vein endothelial cell dysfunction by reducing the levels of inflammatory genes including interleukin-6 (IL-6), macrophage chemoattractant protein-1 (MCP-1), intercellular cell adhesion molecule-1 (ICAM-1), and vascular cell adhesion protein 1 (VCAM-1). ${ }^{12}$ These findings indicated the anti-inflammatory role of irisin. Thus, irisin may be involved in the mechanism of OSAS development and progression through its anti-inflammatory effects.

Skeletal muscles are considered as an endocrine organ that secretes a number of myokines, including follistatin, myostatin, activin A, and irisin. ${ }^{27}$ The present study demonstrated the decreased serum irisin in OSAS patients. However, no investigations have yet focused on the association of OSAS with other myokines. Thus, further studies are necessary to determine the role of other myokines in OSAS.

Serum irisin concentrations were found to be higher in numerous conditions or diseases. Increased circulating irisin was observed in patients with metabolic syndrome, ${ }^{22}$ polycystic ovary syndrome, ${ }^{28}$ and non-alcoholic fatty liver disease. ${ }^{29}$ The increased irisin levels may be attributed to either the increased secretion by adipose/muscle tissue and/or the compensatory irisin increase to overcome an underlying irisin resistance in these subjects.

This study has several potential limitations. First, the sample size was not sufficiently large to arrive at definitive conclusions. Thus, further studies with larger samples are warranted. Second, the cross-sectional design of our study requires its causative relation to be confirmed by future longitudinal studies. Third, we enrolled only male subjects in our study. There are significant gender differences in serum irisin concentrations. Irisin levels were lower in males than females. ${ }^{30,31}$ Therefore, this condition may lead to biased results. We intend to conduct a further study using a population of both male and female subjects.

In conclusion, serum irisin concentrations were negatively correlated with the presence and severity of OSAS.

\section{REFERENCES}

1. Khassawneh B, Ghazzawi M, Khader Y, et al. Symptoms and risk of obstructive sleep apnea in primary care patients in Jordan. Sleep Breath. 2009;13:227-232.

2. Young T, Palta M, Dempsey J, Skatrud J, Weber S, Badr S. The occurrence of sleep-disordered breathing among middle-aged adults. $N$ Engl J Med. 1993;328:1230-1235.

3. Punjabi NM, Newman AB, Young TB, Resnick HE, Sanders MH. Sleep-disordered breathing and cardiovascular disease: an outcome-based definition of hypopneas. Am J Respir Crit Care Med. 2008;177:1150-1155.

4. Young T, Skatrud J, Peppard PE. Risk factors for obstructive sleep apnea in adults. JAMA. 2004;291:2013-2016.

5. Pillar G, Shehadeh N. Abdominal fat and sleep apnea: the chicken or the egg? Diabetes Care. 2008;31:S303-S309.

6. Aihara K, Oga T, Chihara Y, et al. Analysis of systemic and airway inflammation in obstructive sleep apnea. Sleep Breath. 2013;17:597-604. 
7. Moreno-Navarrete JM, Ortega F, Serrano M, et al. Irisin is expressed and produced by human muscle and adipose tissue in association with obesity and insulin resistance. J Clin Endocrinol Metab. 2013;98:E769-E778.

8. Roca-Rivada A, Castelao C, Senin LL, et al. FNDC5/irisin is not only a myokine but also an adipokine. PLoS ONE. 2013;8:e60563.

9. Boström P, Wu J, Jedrychowski MP, et al. A PGC1-a-dependent myokine that drives brown-fat-like development of white fat and thermogenesis. Nature. 2012;481:463-468.

10. Lee P, Linderman JD, Smith S, et al. Irisin and FGF21 are cold-induced endocrine activators of brown fat function in humans. Cell Metab. 2014;19:302-309.

11. Polyzos SA, Kountouras J, Anastasilakis AD, Geladari EV, Mantzoros CS. Irisin in patients with nonalcoholic fatty liver disease. Metabolism. 2014;63:207-217.

12. Zhang Y, Mu Q, Zhou Z, et al. Protective effect of Irisin on atherosclerosis via suppressing oxidized low density lipoprotein induced vascular inflammation and endothelial dysfunction. PLoS ONE. 2016;11:e0158038.

13. American Academy of Sleep Medicine. European Respiratory Society. Australasian Sleep Association. American Thoracic Society Sleeprelated breathing disorders in adults: recommendations for syndrome definition and measurement techniques in clinical research: the report of an American Academy of Sleep Medicine Task Force. Sleep. 1999;22:667-689.

14. Durán J, Esnaola S, Rubio R, Iztueta A. Obstructive sleep apneahypopnea and related clinical features in a population-based sample of subjects aged 30 to $70 \mathrm{yr}$. Am J Respir Crit Care Med. 2001;163:685-689.

15. Peppard PE, Young T, Palta M, Dempsey J, Skatrud J. Longitudinal study of moderate weight change and sleep-disordered breathing. JAMA. 2000;284:3015-3021.

16. Romero-Corral A, Caples SM, Lopez-Jimenez F, Somers VK. Interactions between obesity and obstructive sleep apnea: implications for treatment. Chest. 2010;137:711-719.

17. Schwab RJ, Pasirstein M, Pierson R, et al. Identification of upper airway anatomic risk factors for obstructive sleep apnea with volumetric magnetic resonance imaging. Am J Respir Crit Care Med. 2003;168:522-530.

18. Patel SR, Larkin EK, Redline S. Shared genetic basis for obstructive sleep apnea and adiposity measures. Int J Obes (Lond). 2008;32:795-800.
19. Huh JY, Dincer F, Mesfum E, Mantzoros CS. Irisin stimulates muscle growth-related genes and regulates adipocyte differentiation and metabolism in humans. Int J Obes (Lond). 2014;38:1538-1544.

20. Blüher S, Panagiotou G, Petroff D, et al. Effects of a 1-year exercise and lifestyle intervention on irisin, adipokines, and inflammatory markers in obese children. Obesity (Silver Spring). 2014;22:1701-1708.

21. Hou N, Han F, Sun $X$. The relationship between circulating irisin levels and endothelial function in lean and obese subjects. Clin Endocrinol (Oxf). 2015;83:339-343.

22. Park KH, Zaichenko L, Brinkoetter M, et al. Circulating irisin in relation to insulin resistance and the metabolic syndrome. J Clin Endocrinol Metab. 2013;98:4899-4907.

23. Liu JJ, Wong MD, Toy WC, et al. Lower circulating irisin is associated with type 2 diabetes mellitus. J Diabetes Complications. 2013;27:365-369.

24. Huh JY, Panagiotou G, Mougios V, et al. FNDC5 and irisin in humans: I. Predictors of circulating concentrations in serum and plasma and II. mRNA expression and circulating concentrations in response to weight loss and exercise. Metabolism. 2012;61:1725-1738.

25. Bosma M, Gerling M, Pasto J, et al. FNDC4 acts as an antiinflammatory factor on macrophages and improves colitis in mice. Nat Commun. 2016;7:11314.

26. Wang C, Wang L, Liu J, et al. Irisin modulates the association of interleukin-17A with the presence of non-proliferative diabetic retinopathy in patients with type 2 diabetes. Endocrine. 2016;53:459-464.

27. Vamvini MT, Aronis KN, Panagiotou G, et al. Irisin mRNA and circulating levels in relation to other myokines in healthy and morbidly obese humans. Eur J Endocrinol. 2013;169:829-834.

28. Bostancı MS, Akdemir N, Cinemre B, Cevrioglu AS, Özden S, Ünal O. Serum irisin levels in patients with polycystic ovary syndrome. Eur Rev Med Pharmacol Sci. 2015;19:4462-4468.

29. Choi ES, Kim MK, Song MK, et al. Association between serum irisin levels and non-alcoholic fatty liver disease in health screen examinees. PLoS ONE. 2014;9:e110680.

30. Anastasilakis AD, Polyzos SA, Saridakis ZG, et al. Circulating irisin in healthy, young individuals: day-night rhythm, effects of food intake and exercise, and associations with gender, physical activity, diet, and body composition. J Clin Endocrinol Metab. 2014;99:3247-3255.

31. Al-Daghri NM, Alkharfy KM, Rahman S, et al. Irisin as a predictor of glucose metabolism in children: sexually dimorphic effects. Eur J Clin Invest. 2014;44:119-124. 